\title{
Auricular Prosthesis for Congenitally Deformed Ear with Acrylic Template for Colour Depiction - A Case Report
}

\author{
Almas Shaikh ${ }^{1}$, Aushili Mahule ${ }^{2}$, Divyakshi Motwani ${ }^{3}$, Ashwini Shirbhate ${ }^{4}$, Shakun Saraf 5
}

\author{
${ }^{1}$ Department of Prosthodontics, Government Dental College, Mumbai, Maharashtra, India. ${ }^{2}$ \\ Department of Prosthodontics, Crown Bridge and Oral Implantology, Swargiya Dadasaheb Kalmegh \\ Smruti Dental College and Hospital, Nagpur, Maharashtra, India. ${ }^{3}$ DY Patil Dental College, Mumbai, \\ Maharashtra, India. ${ }^{4}$ Vidarbha Youth Welfare Society's Dental College and Hospital, Amravati, \\ Maharashtra, India. ${ }^{5}$ Department of Prosthodontics and Crown and Bridges, Rajesh Ramdasji \\ Kambe Dental College, Akola. Maharashtra, India.
}

\section{INTRODUCTION}

Prosthodontists usually stick to the dictum by MM Devan, "preservation of what remains than meticulous replacement of what has been lost.1" "Microtia" is a term used to describe congenital anomaly of the external ear. It is a combination of the words "micro" and "otia" each of the term denoting small and ear respectively. It includes a range of deformities which may consist of presence of a rudimentary ear, a grossly normal or smaller ear or complete absence of the entire external ear. These deformities usually account for 3 in every ten thousand births, with less than $10 \%$ of all the cases showing bilaterally missing ears. ${ }^{2-4}$ Facial deformities are common for the microtia patient as the auricle develops from tissues of the branchial arches. Figure 1 describes the patient having congenitally missing unilateral ear.

Maxillofacial prosthodontics deals with prosthetic rehabilitation of disfigured or missing parts of head and neck. Prosthetic replacement of the exterior part (Epithesis) can be related to as old as civilization. References to it are available in Indian, Greek, Roman, Egyptian Civilizations. ${ }^{5}$ Ambroise Pare is credited with making various contributions to the materials and techniques in facial prosthetics. Fabrication of an extra-oral prosthesis is probably more of an art than science.

Throughout the recorded history, humans have attempted to restore missing parts of the body by using various artificial materials. MPF materials have evolved since centuries right from metals, ivory, porcelain, waxes, natural rubber, gelatin, and latex to modern day materials such as methacrylate or acrylic resins, polyurethane elastomers, and silicone elastomers each having their own set of advantages and disadvantages. But silicone is most commonly used because of its assorted benefits over other MFP materials. This case report describes an auricular prosthesis for the patient with congenital ear deformity using an acrylic template for colour depiction and room temperature volcanizing (RTV) silicone. ${ }^{6}$

\section{PRESENTATION OF CASE}

A 38-year-old male patient with unilateral congenital defect in his right ear reported to the Department of Prosthetic Dentistry, GDC and hospital, for rehabilitation. Clinical examination and history revealed the presence of some congenitally present rudimentary ear near the tragus region of missing ear (Figure 1). Hearing capability was normal on both the sides. No history of any surgical attempt to correct the deformity or any previous prosthesis was given. Patient was offered the surgical treatment options to which he did not agree. Thus, prosthetic rehabilitation was planned without any surgical intervention. Before carrying out any procedures, patient was counselled and educated regarding the nature, function as well as limitation of the prosthesis. An informed written consent was made. For assessment and evaluation, pre-operative photographs were taken.
Corresponding Author: Dr. Aushili Mahule, Post Graduate Student, Department of Prosthodontics, Crown Bridge and Oral Implantology, Swargiya Dadasaheb Kalmegh Smruti Dental College and Hospital, Nagpur, India. E-mail:aush.mahule@gmail.com

DOI: $10.14260 /$ jemds/2021/624

How to Cite This Article:

Shaikh A, Mahule A, Motwani D, et al. Auricular prosthesis for congenitally deformed ear with acrylic template for colour depiction - a case report. J Evolution Med Dent Sci 2021;10(35):3063-3066, DOI: 10.14260/jemds/2021/624

Submission 20-05-2021,

Peer Review 15-07-2021,

Acceptance 22-07-202,

Published 30-08-2021.

Copyright (c) 2021 Almas Shaikh et al. This is an open access article distributed under Creative Commons Attribution License [Attribution 4.0 International (CC BY 4.0)] 


\section{DISCUSSION OF MANAGEMENT}

The patient was draped and protected by surgical cap keeping exposed only the rudimentary ear and a small area surrounding it. ${ }^{7}$ In order to prevent entry of the impression material, external auditory canal was blocked with gauze. Vaseline was carefully applied to the operating area and the skin surrounding it. Impressions of the auricular defect were made using irreversible hydrocolloid, (Neocolloid, Dental Products India) following standard procedures. The impression was then poured with Type IV die material and Type III dental stone, using the standard procedures. ${ }^{8} \mathrm{~A}$ caliper was used to measure the patient's normal ear dimension that included the entire supero-inferior and anteroposterior dimensions. The helical, antihelical pattern and shape, concha as well as the lobule and skin colour of the ear were carefully observed to aid in fabrication of the wax pattern and for shade matching. Detailed photography was done. The other normal ear of the patient was used as the donor ear.
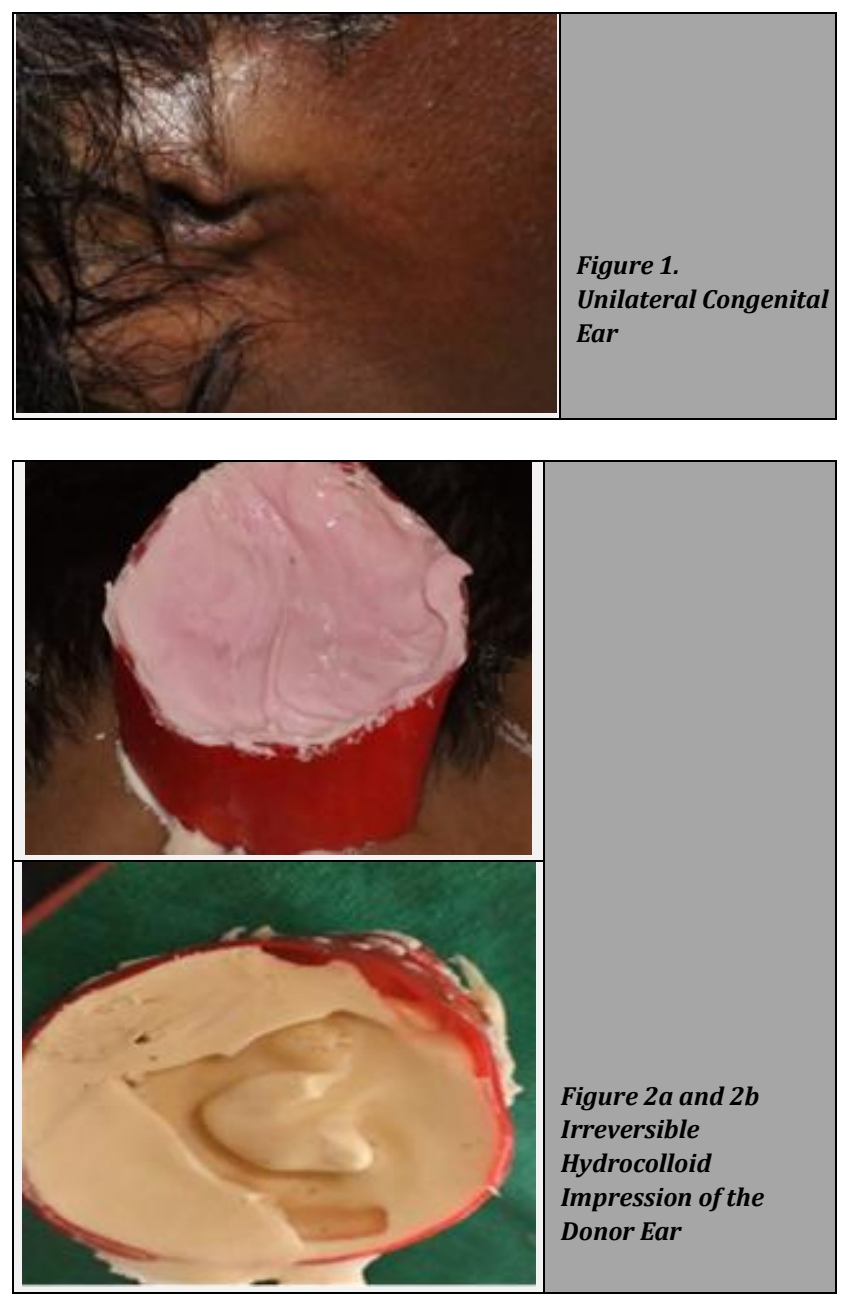

An irreversible hydrocolloid impression of the donor ear was made and the cast was poured (Figure 2a \& 2b). Keeping the contralateral ear model as a reference, free hand wax pattern was carved, with an attempt to simulate the same as far as possible. ${ }^{9}$ The wax pattern was tried on the patient's face and a thorough assessment was made both from frontal as well as the profile views. Modifications required were done and final finishing of the wax pattern was performed. An irreversible hydrocolloid impression of the wax pattern was made, a gypsum cast was poured and a vacuum formed template for shade matching was made from the cast and was checked for adaption over wax pattern (Figure 3a \& 3b).
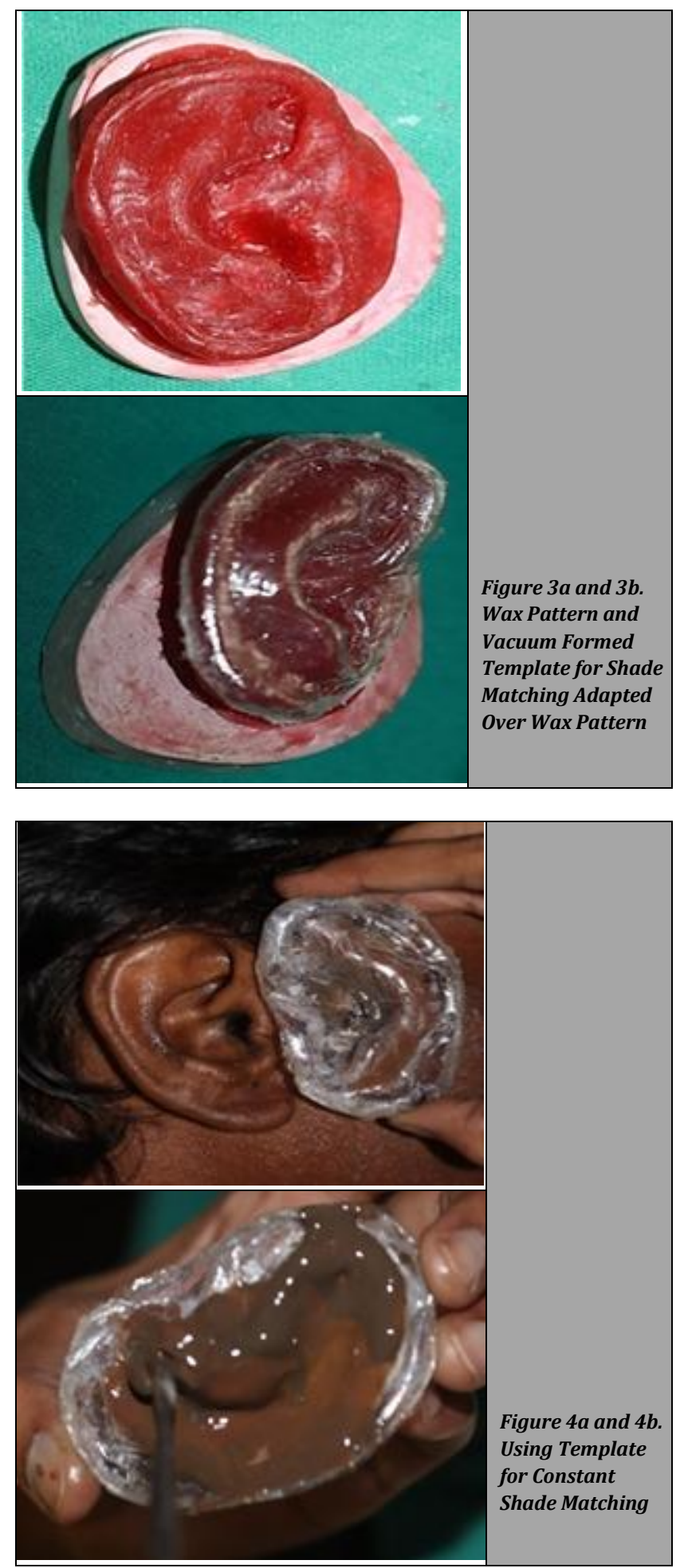

The finished wax pattern was then sealed on the stone cast. A three-part flasking was done using sodium alginate as a separating medium. Using standard procedures, dewaxing was carried out in a hot water bath.

Shade selection was carried out. Yellow, white, brown, purple, and red were used as basic shades of colour. Intrinsic stains as well as extrinsic staining (Technovent) with the room temperature vulcanizing silicone (MDX-4, RTV) were used for shade matching. Base and catalyst pastes were taken in 
appropriate amount and mixed and increments were added over the template itself which provided an easier method for shade matching with the surrounding skin, providing optimal results. Instead of the previous one which used dewaxed mould for silicone mixing and packing, the use of template provided better results as constant shade matching was easily done (Figure 4a \& 4b). Different shades were used to accurately replicate various parts of the patient's natural ear and provide a life-like prosthesis. ${ }^{10}$ Different sources of lights were used (incandescent, fluorescent, and natural sun light sources) to achieve proper shade matching.

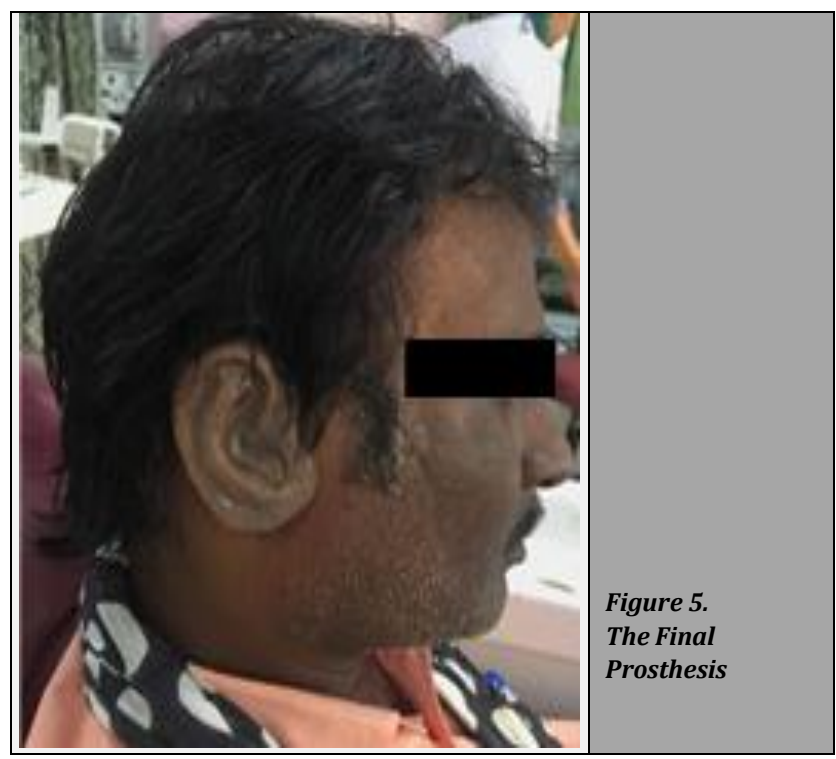

The room temperature silicon material was packed and the three-part flasking was carefully re-seated to ensure proper flushing of the margins. According to the manufacturer's instructions the flash was kept for bench press for 48 hours prior to opening the flask. The silicone prosthesis was then examined for defects and porosities, trimmed and finished. The final prosthesis was tried on the patient (Figure 5). Retentive aid used was a bio-adhesive (G609 Probond Adhesive). ${ }^{11}$ Post-operative instructions were given and regular follow up was carried out.

\section{DISCUSSION}

Patients with facial deformities endure psychological affliction. The aim of maxillofacial rehabilitation is to provide a suitable prosthesis for patients with facial defects so that they can be confident enough to face the society and accept the challenges of life. Auricular defects can be repaired or reconstructed with autogenous tissue, but surgical interventions may not be a treatment of choice most of the time due to personal / medical reasons. At times, surgical reconstruction may vary and present certain challenges and limitations both for the clinician as well as the patients. A simple procedure for rehabilitation of the missing ear is presented in this article. Prosthetic replacement offers assorted advantages over surgical reconstruction, such as being cost effective, aesthetically acceptable for patients and allowing for periodic evaluation and cleaning of the surgical site. 12 The fabrication process is also relatively short, and the clinician can have a good control over the colour, shape, and position of the prosthesis. Additionally, prosthetic restoration is reversible in contrast to the reconstructive surgery.

A prosthetic ear is an artificial ear that is usually made of silicone from a mould that is prepared using the opposite ear (or a parent's ear in bilateral cases) as a template. Prosthetic ear provides cosmetic reconstruction, aids to direct the sound waves into the auditory canal and also maintains a proper environment for the inner ear membranes. The ear prosthesis may also help to retain eyeglasses and a hearing aid, if needed. ${ }^{13}$

Several MFP materials are available nowadays. In this case, RTV silicone (MDX-4, RTV) was used. Silicones are more preferred as they are biocompatible, flexible, comparatively cost effective, light-weighted, and provide cosmetically elegant (life-like appearance) to prosthesis. Medical-grade silicone material is colour stable and can match with any skin colour by using intrinsic and extrinsic colour systems. Intrinsic stains were used for colouring the prosthesis as they provide more colour stability and better esthetics. ${ }^{14}$ Extrinsic staining was done as needed.

Silicones have certain disadvantages like they are difficult to conceal the skin-prosthesis margin, difficulty in shade matching, a need for regular replacement and / or repair, and more precautions are necessary during removal of the prosthesis whenever the person is involved in rigorous activity. Also, material contact with sweat, dust, pollen, and hot, humid conditions causes gradual discoloration the hardening process of silicone over a period of time, but the material still remains in considerably acceptable condition for about 9-12 months of time.

Various means of retention for MFP are available such as bio-adhesives, magnets, clips or the prosthesis can be attached to the spectacle. But skin adhesives are cost effective, easy to use, adhere well under moist conditions and hence most common aid of retention for MFP. In this case, bio-adhesive G609 Probond Adhesive (Technovent, Bridgend) was used for retention of the prosthesis. It's a water based adhesive and provided sufficient retention for the prosthesis. It consisted of aqueous dispersion of acrylate copolymer. The adhesives can be cleaned with simple soap and water. Water-based adhesives are milder but do not adhere as long as siliconebased adhesives as they are not moisture resistant. However, the disadvantage is skin adhesives may sometimes lead to allergic contact dermatitis. ${ }^{11,15}$

Treatment was patient centred in this case. The rudimentary ear tissues were used in combination with a bioadhesive which provided a very non-invasive approach to fabricate a maxillofacial prosthesis. The implant supported prosthesis if used would have provided a better retention, stability and comfort of the prosthesis. However, the patient was not ready for a surgical treatment modality and hence the mentioned treatment plan remained best suited.

Also, a point of consideration is that, treating a unilateral maxillofacial prosthesis case is a more challenging task as compared to bilaterally involved cases because there is a constant comparison with a natural counterpart.

Recent advances for treating maxillofacial cases include a production of auricular prosthesis having mirror image using computed tomography scanner and three-dimensional systems, alleviating most of the limitations of conventional prosthesis. However, high-cost limits its use contemporarily. 
Development in the field of tissue engineering in near future will augment the outcome of prosthodontic rehabilitation.

\section{CONCLUSIONS}

This article described a simple yet aesthetically, functionally and economically fulfilling technique to rehabilitate patients with auricular defects. It also helped the patients to restore to psychological normalcy, prevented them from further social stigma without involving any surgical intervention. It provided a psychological benefit to the patient's physical, mental wellbeing.

Financial or other competing interests: None.

Disclosure forms provided by the authors are available with the full text of this article at jemds.com.

\section{REFERENCES}

[1] DeVan MM. The nature of the partial denture foundation: suggestions for its preservation. Journal of Prosthetic Dentistry 1952;2(2):210-8.

[2] Krishnan VCJ, Balaji SM, Jain AR. A simple ear splint for microtia patients. Indian Journal of Dental Research 2015;26(2):220-1.

[3] Wolfaardt JF, Coss P. An impression and cast construction technique for implant-retained auricular prostheses. Journal of Prosthetic Dentistry 1996;75(1):45-9.

[4] Cheng AC, Morrison D, Cho RS, et al. Vacuum-formed matrix as a guide for the fabrication of craniofacial implant tissue bar-retained auricular prostheses. Journal of Prosthetic Dentistry 1998;79(6):711-4.

[5] Mantri SS, Thombre RU, Pallavi D. Prosthodontic rehabilitation of a patient with bilateral auricular deformity. J Adv Prosthodont 2011;3(2):101-5.
[6] De Caxias FP, Dos Santos DM, Bannwart LC, et al. Classification, history and future prospects of maxillofacial prosthesis. International Journal of Dentistry 2019;2019:8657619.

[7] Kubon TM, Kurtz KS, Piro JD. Impression procedure for creating a partial auricular prosthesis. J Prosthet Dent 2000;83(6):648-51.

[8] Wang R. Preoperative auricular wax pattern duplication for surgical template fabrication. J Prosthet Dent 1999;81(5):634-7.

[9] Al Mardini M, Ercoli C, Graser GN. A technique to produce a mirror-image wax pattern of an ear using rapid prototyping technology. J Prosthet Dent 2005;94(2):195-8.

[10] Goiato MC, Haddad MF, Sinhoreti MAC, et al. Influence of opacifiers on dimensional stability and detail reproduction of maxillofacial silicone elastomer. Biomed Eng Online 2010;9:85.

[11] Kiat-Amnuay S, Gettleman L, Khan Z, et al. Effect of adhesive retention on maxillofacial prostheses. Part I: skin dressings and solvent removers. J Prosthet Dent 2000;84(3):335-40.

[12] Naveen BH, Kashinath KR, Shankargouda SB, et al. A blue tooth-auricular prosthesis: a case report. Journal of Clinical and Diagnostic Research 2014;8(8):ZD36-8.

[13] Chinnasamy A, Gopinath V, Jain AR. Ear prosthesis for postburn deformity. Case Reports in Otolaryngology 2018;2018:2689098.

[14] Andres CJ, Haugh SP. Facial prosthesis fabrication: technical aspects. In: Taylor TD, edr. Clinical maxillofacial prosthetics. Illinois: Quintessence Publishing Co, Inc., 2000.

[15] Parel SM. Diminishing dependence on adhesives for retention of facial prostheses. J Prosthet Dent 1980;43(5):552-60. 